\title{
A Review on IOT Based Smart Home Automation Using Renewable Energy Sources
}

\author{
Dipali D. Ghorpade ${ }^{1}$, A. M. Patki ${ }^{2}$ \\ ${ }^{1}$ Final Year M.E Communication Systems, ICEM, Pune \\ ${ }^{2}$ Professor and HOD of Electronics and Telecommunication, ICEM, Pune
}

\begin{abstract}
Smart Home architecture designed with the help of Internet of Things. We propose a monitoring and Remote control of domestic equipments from an Android application based on Raspberry pi card. To minimize the amount of carbon emissions that we contribute towards the cumulative carbon emissions of this earth, Use of Renewable Energy Sources in Household application has always been the most effective method. By developing different codes the communication between the remote user, the web server, the raspberry pi card and the home components is possible.
\end{abstract}

Keywords: Internet of Things(IoT), raspberry pi, renewable energy sources, electronic components, web server

\section{Introduction}

Now a days Carbon emission of the planet earth has been increased in a large extent due to industrialization, automation, modern life of the people. Use of non-renewable energy sources is very much dependent for it, which has given rise to global warming due to depletion of ozone layer. Hence use of renewable energy sources is very much effective method to minimize the amount of carbon emission. Now use of renewable energy sources like solar energy is very much applicable to the home automation[4].Home automation includes all electronic components, subsystems of the house and company such as heating, garage door, entrance gate, shutters, electronic outlets etc. to meet the comfort needs such that energy management \& optimization of lighting \& heating home automation has been developed to provide technical solutions. Hence improved through a use of communication network that includes a pair of twisted lines, fibre optics in a bus based network or an internet protocol as standards. The device technology \& communication technology has been rapidly developed offers the facilities to development of the electronic systems [1].In the large area of application i.e. monitoring and control in the home automation, industry, water monitoring, health monitoring etc. data acquisition plays very important role. Recent years there is requirement to do the long distance monitoring. Hence remote monitoring based on web is known as internet of things (IOT) framework is a choice. In this work has been designed, implemented to control \&monitoring of household equipment's based on IOT, where the data monitoring is possible by web browser \& can be access via web browser devices i.e. computer, laptop or small mobile phone[2].The proposed system designed and implemented a low cost credit card sized raspberry can be control through internet under the android environment, household equipment such as heating, water tank level motioning, air conditioning , gas sensing etc. Application consists to develop programs that allow to communication between a remote user using a smart phone, web browser and raspberry pi card that communicate with one or more interface cards to control the Equipment in the home using solar energy. This would eventually put an impact on the total carbon emission due to generation process of power from non-renewable energy sources.

The paper organized by as follows. Section 2 is a proposed system overview which introduces the concept of IoT in which we describe different hardware components providing internet connectivity support for data acquisition from electric equipment and communication within modules are described. Section 3 discusses the scalability and sustainability of the whole project. Finally we conclude our outcome in section 4 .

\section{Proposed System Overview}

IoT is nothing but the internet of things in which where daily life all things connected to internet and can be monitor $\&$ can be operate remotely from anywhere.

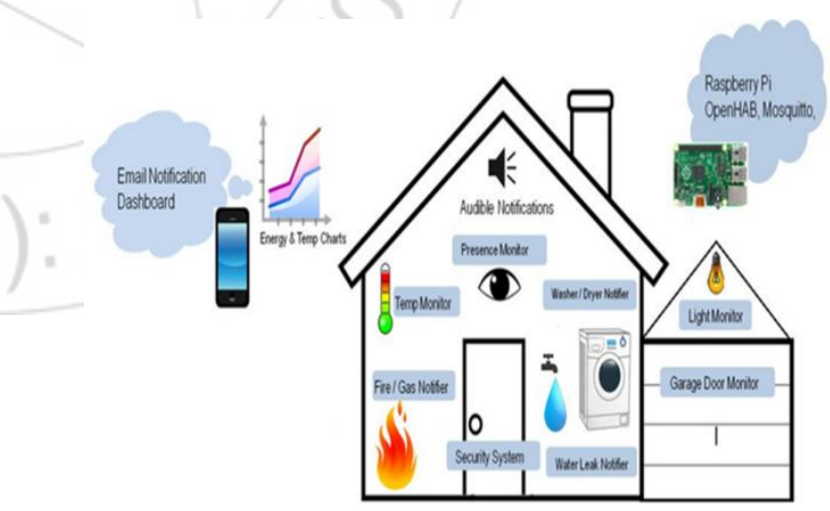

Figure 1: Smart Home Architecture

Daily life things like green agriculture, environmental monitoring, industrial wireless sensor network or industrial management, urban management, Tele-medicine, intelligent transportation and smart homes etc. IoT devices can be used to monitor and control the electrical and electronic system used in various types of buildings (e.g Industrial, Institutions or residential).As shown in fig.1 Home automation systems are typically used to control ventilation, air conditioning, lighting, heating, appliances, entertainment and home security devices, communication systems to 


\section{International Journal of Science and Research (IJSR) \\ ISSN (Online): 2319-7064}

Index Copernicus Value (2013): 6.14 | Impact Factor (2015): 6.391

improve comfort, security and energy efficiency.Here we are designing solar based home automation, where all equipment's i.e. tank water level indicator, exact position of sun tracking solar panels, Entrance Door indicator etc. are interface with Raspberry pi Board. And this information of home automation, owner directly monitors and control through their mobile phone using IOT (Internet of Things).

Here in this application all gadgets are controlled by Solar panels power we are designed first how much power taking by all equipment's, because of that we are constructing a sun tracking system using 3 LDR sensors, through LDR sensor Raspberry pi knows exact position of sun so solar panel are also rotates through DC motor based tracker.

For tank water level indicator, we are using Float sensor placed in tank and measure water level. Depending upon water level i.e.lesser than set point water motor automatically ON from Raspberry pi cardthrough Relay.

If there is any leakage in gas pipe it will also automatically indicating through sensor controlling Raspberry Pi. And also send the message to the owner to take action on it.Also Here we are using entrance door indicator so raspberry pi assemble with ultrasonic sensor if any one come close to the entrance door alarm will be $\mathrm{ON}$ automatically. And also these all information owners automatically monitor/and control from his Mobile phone, i.e. if anyone come closer to the door, Camera will detect the object and show on mobile phones of owner through IOT. And if owner knows the visitor so through mobile phone he opens the door from giving command from mobile thought IOT only.

\section{Scalability}

The setup can be configured with the electrical components being chosen according to the scale of the project. It can be a low cost installation on a rural home and can be extended to a high security setup for bigger projects. As it is easy to set up on existing homes with modern electronic equipment's installed, it is very scalable. As the services are provided through the Internet of things, the procedure of operation can be remotely reconfigured depending on needs and user feedback. Time to time when the need arises, the web services can be reconfigured.

\section{Conclusion}

Due to easy implementation the designed system is very customizable according to needs. Use of our renewable energy resources which would otherwise have been underutilized is possible by this effective technique. The remote control includes software and hardware components such as web server, smart phone, Raspberry pi card and interfacing sensors. By developing and storing many codes in the web server, smart phone and the raspberry pi card, we can monitor and get remote command of multiple domestic equipments. The integration of Internet of Things with existing smart home architecture, it may be more autonomous, more practice, and quite scalable saw the giant step and progress in the areas of technology and communication in our time .It will provide us numerous opportunities for improvements in our energy saving techniques.

\section{References}

[1] Hayet Lamine, Hafedh Abid "Remote control of a domestic equipment from an Android application based on Raspberry pi card," in 15th international conference on Sciences and Techniques of Automatic control \& computer engineering - STA'2014, Hammamet, Tunisia, December 21-23, 2014 @2014 IEEE

[2] M. F.AL. Faisal, S Bakar, PS Rudati, "The Development of A Data Acquisition System Based on Internet of Things Framework",@IEEE.

[3] Raguvaran. K, Mr. J. Thiyagarajan, M.E., (Ph.D.), "Raspberry PI Based Global Industrial Process Monitoring Through Wireless Communications" International Conference on Robotics, Automation, Control and Embedded Systems - RACE 2015 18-20 February 2015, Hindustan University, Chennai, India.

[4] Saswat Mohanty, Bikash Narayan Panda, Bhawani Shankar Pattnaik" Implementation of a Web of Things based Smart Grid to remotely monitor and control Renewable Energy Sources"2014 IEEE Students' Conference on Electrical, Electronics and Computer Science

[5] Y. Fan, Y. Yin, L. Xu, Y. Zeng, and F. Wu, "IoT based smart rehabilitation system," IEEE Transactions on Industrial. Informatics, vol. 10, no. 2, pp. 1568-1577, 2014.

[6] Md. Nasimuzzaman Chowdhury, Md. Shiblee Nooman and Srijon Sarker, "Access Control of Door and Home Security by Raspberry Pi through Internet," IJSER, vol. 4, issue. 11, pp. 550-558, 2013.

[7] D. Ibrahim, Raspberry Pi Hardware Project, Elektro International Media, p.9, 2014

[8] Mukesh Kumar, Sanjeev Sharma, and Mansav Joshi, "Design of Real Time Data Acquisition with Multi Node Embedded Systems," IJCA., vol. 42, no. 11, pp. 6-12, 2012. 